\title{
Coupled Flare Attractors - A Discrete Prototype for Economic Modelling
}

\author{
GEORG C. HARTMANN * and OTTO E. ROSSLER \\ Division of Theoretical Chemistry, Auf der Morgenstelle 8, University of Tübingen, 72076 Tübingen, FRG
}

(Received in final form 7 January 1998)

\begin{abstract}
A chaotic environment can give rise to "flares" if an autocatalytic variable responds in a multiplicative, threshold-type fashion to the environmental forcing. An "economic unit" similarly depends in its growth behavior on the unpredictable (chaotic?) buying habits of its customers, say. It turns out that coupled flare attractors are surprisingly robust in the sense that the resulting "economy" is largely independent of the extent of diffusive coupling used. Some simulations are presented.
\end{abstract}

Keywords: Mathematical economics, Singular-continuous-nowhere-differentiable attractors, Kaplan-Yorke attractors, Flares, Diffusive coupling

\section{INTRODUCTION}

Chaos by definition is non-robust. The butterflyeffect [1,2], as featured in Steven Spielberg's blockbuster movie "Jurassic Park", is the bestknown example perhaps. Flare attractors reflect this unpredictability and amplify it. This is because only certain symbolic dynamic sequences - many consecutive "ones" rather than an even mixture of zeros and ones, say (that is, many consecutive suprathreshold rather than subthreshold chaotic inputs) - support an extended period of autocatalytic growth (a flare). One would therefore expect these attractors to be very sensitive to environmental influences. Unexpectedly, this is not the case.
As we shall see in the following, many coupled flare attractors do not strongly influence each other. Therefore, they can be used to generate an abstract "model economy" in the computer.

\section{AN EQUATION}

Figure 1 illustrates the principle. A corresponding discrete equation is, for example,

$$
\begin{aligned}
& x_{n+1}=4 x_{n}\left(1-x_{n}\right), \\
& b_{n+1}=b_{n}+b_{n}\left(x_{n}-\text { threshold }\right)-\epsilon b_{n}^{2} .
\end{aligned}
$$

Here, the first variable $(x)$ is the well-known logistic map [3]. The second variable $(b)$ grows

\footnotetext{
* Corresponding author.
} 
autocatalytically whenever $x_{n}$, the momentary value of the chaotic forcing, exceeds the threshold value assumed. The small parameter $\epsilon>0$ prevents the second variable from reaching unrealistic unbounded flare amplitudes. Figure 2 shows a simulation.

Figure 2(a) is self-explanatory: The name "flares" is directly applicable to the elements of

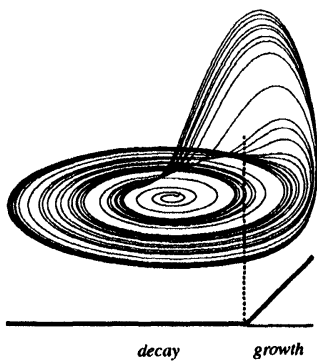

FIGURE 1 Basic mode of action of a flare attractor. A chaotic subsystem "forces" a nonlinearly responding autocatalytic unit (schematic drawing).
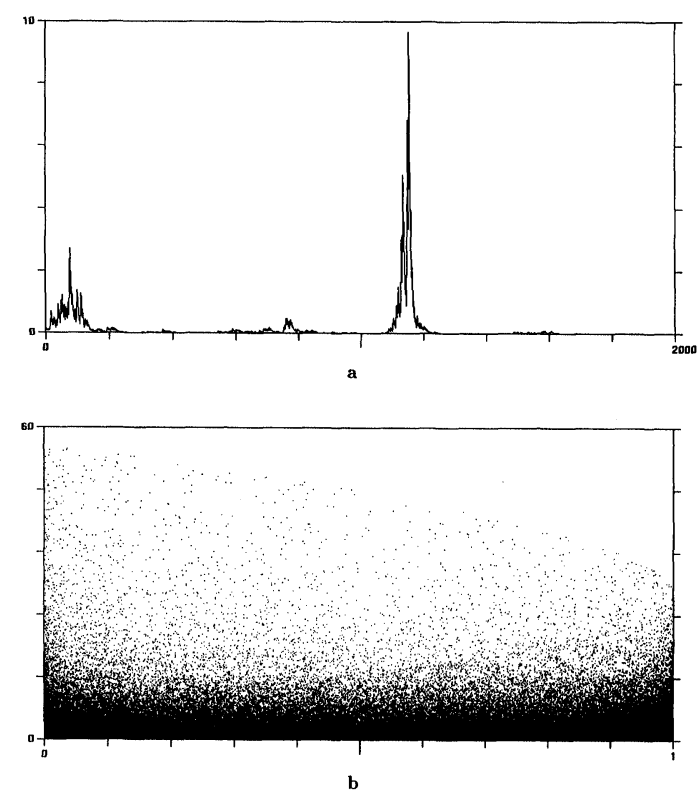

FIGURE 2 A simple flare attractor based on the logistic difference equation: Numerical simulation of Eq. (1). (a) Time plot of the flaring variable, $b$. Hereby successive points were connected by a straight line segment. (b) Side view $(x, b$ plot). Parameter values: threshold $=0.7, \epsilon=0.01$. Initial conditions: $x_{1}=0, b_{1}=1$. Iteration number: 2000 for (a); and 1000000 for (b). This and all following calculations were done at 16digit precision. such a time series. The $x, b$ plot (Fig. 2(b)) is also characteristic: If one waits long enough, a screen-filling black "curtain" is eventually obtained. In the transient picture shown here, the exponentially decreasing density, towards the top of the attractor, makes itself manifest to the eye.

For curiosity's sake, we also present, in Fig. 3, a more sophisticated flare attractor. It is generated
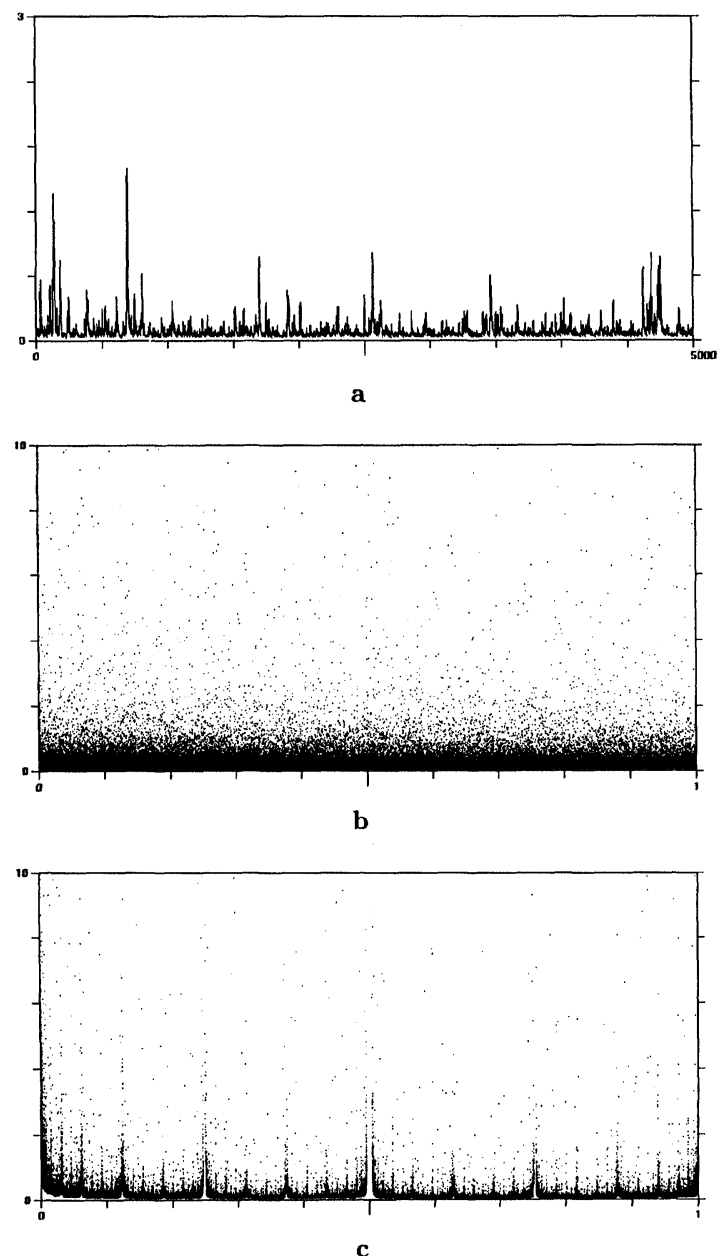

FIGURE 3 Flare attractor generated by an invertible map, Eq. (2). (a) Time behavior as in Fig. 2(a), but longer. (b) Side view $(x, b$ plot) as in Fig. 2(b). (c) Cross-view $(y, b$ plot). A cross section between $x=0$ and $x=1$ is shown (note that no narrower slice is necessary with this particular map). 1000000 iterations are shown (in (b) and (c)). Initial conditions: $x_{0}=\frac{1}{10} \sqrt{2}, y_{0}=0.1, b_{0}=0.1, t_{\text {end }}=5000$ (in (a)). 
by an invertible three-variable map:

$$
\begin{aligned}
& x_{n+1}= \begin{cases}\left(2-10^{-15}\right)-2 x_{n} & \text { if } x_{n} \leq \frac{1}{2}, \\
2 x_{n} & \text { if } x_{n}>\frac{1}{2},\end{cases} \\
& y_{n+1}= \begin{cases}1-\left(\frac{1}{2}-0.05\right) y_{n} & \text { if } x_{n} \leq \frac{1}{2}, \\
\left(\frac{1}{2}-0.05\right) y_{n} & \text { if } x_{n}>\frac{1}{2},\end{cases} \\
& b_{n+1}=b_{n}+b_{n}\left(0.37-x_{n}\right)-10^{-3} z_{n}^{2}+10^{-2} .
\end{aligned}
$$

The first two variables here jointly form the "tent" baker's map [4], although so with some contraction due to the small constant $(0.05)$ subtracted from the factor $\frac{1}{2}$ in the same line. (This contraction assures genericity for the forcing attractor.) The first two pictures clearly closely resemble those of Fig. 2. The third picture, however, the $y, b$ plot of Fig. 3(c), shows a cross section through the attractor which is generated by this invertible map. One sees a self-similar fractal with gaps the "lion's paw" as it has been called [5]. Note that in this flare attractor, the sign of the product term containing the threshold has been inverted compared to Eq. (1). If instead the convention of Eq. (1) had been used, the lion's paw would be replaced by the "fiery flames fractal" [5]. These invertible flare attractors are examples of singular-continuous-nowhere-differentiable (SCND) attractors, cf. [6-8].

Obviously, the flaring behavior of the third variable is largely independent of the intrinsic complexity of the forcing chaotic subsystem.

\section{COUPLED FLARE ATTRACTORS}

Figure 4 shows the sum dynamics of several flare attractors - first of three, then of six, finally of 18 of them. The difference equations used to generate these pictures were:

$x_{n+1}^{(1)}=3.99 x_{n}^{(1)}\left(1-x_{n}^{(1)}\right)$,

$b_{n+1}^{(1)}=b_{n}^{(1)}+b_{n}^{(1)}\left(0.565-x_{n}^{(1)}\right)-10^{-3} z_{n}^{(1)^{2}}+10^{-3} s$,

$x_{n+1}^{(2)}=3.99 x_{n}^{(2)}\left(1-x_{n}^{(2)}\right)$,

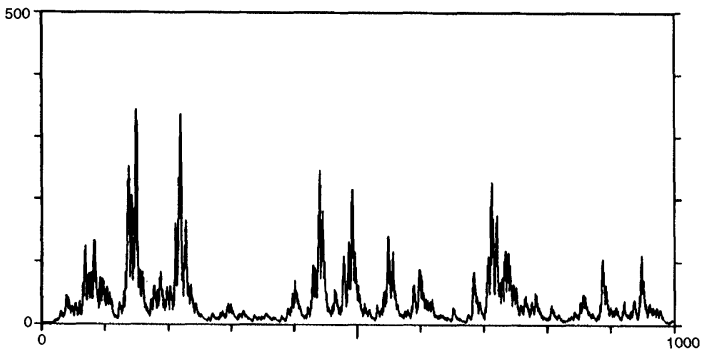

$\mathbf{a}$

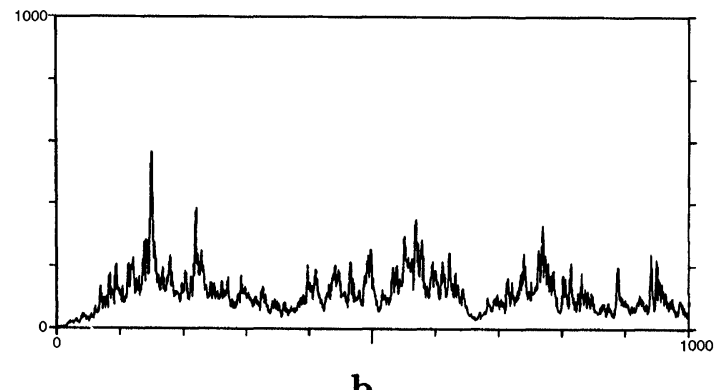

b
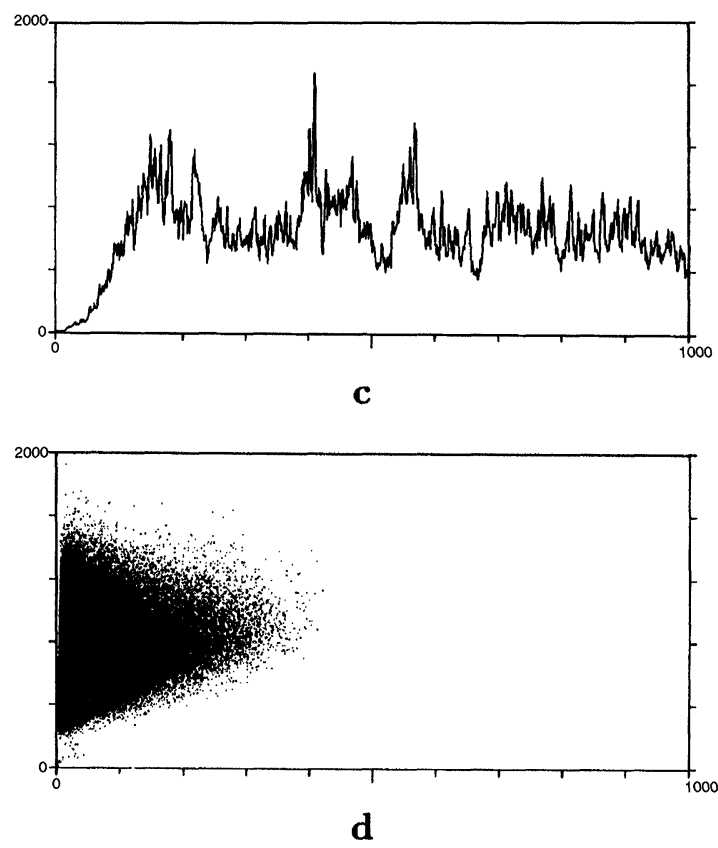

FIGURE 4 Several coupled flare attractors, superposed. Numerical simulation of Eq. (3). (a) Time plot of $b^{(1)}$. A very similar picture was, by the way, obtained if the last term in the second line of Eq. (3) was replaced by a constant, 0.6. (b) Time plot of $b$ (1) through $b^{(6)}$, superposed, i.e. of $s$. (c) Time plot of $b^{(1)}$ through $b^{(18)}$, superposed. (d) $b^{(1)}, s$ plot, 1000000 iterations shown. Initial conditions for the first variables $x^{(j)}: 0.010,0.011$, etc.; for the second variables $b^{(j)}$ : 0.2 . 
$b_{n+1}^{(2)}=b_{n}^{(2)}+b_{n}^{(2)}\left(0.566-x_{n}^{(2)}\right)-10^{-3} z_{n}^{(2)^{2}}+10^{-3} s$,

$x_{n+1}^{(3)}=3.99 x_{n}^{(3)}\left(1-x_{n}^{(3)}\right)$,

$b_{n+1}^{(3)}=b_{n}^{(3)}+b_{n}^{(3)}\left(0.567-x_{n}^{(3)}\right)-10^{-3} z_{n}^{(3)^{2}}+10^{-3} s$,

$x_{n+1}^{(4)}=3.99 x_{n}^{(4)}\left(1-x_{n}^{(4)}\right)$,

$b_{n+1}^{(4)}=b_{n}^{(4)}+b_{n}^{(4)}\left(0.568-x_{n}^{(4)}\right)-10^{-3} z_{n}^{(4)^{2}}+10^{-3} s$,

$x_{n+1}^{(5)}=3.99 x_{n}^{(5)}\left(1-x_{n}^{(5)}\right)$,

$b_{n+1}^{(5)}=b_{n}^{(5)}+b_{n}^{(5)}\left(0.569-x_{n}^{(5)}\right)-10^{-3} z_{n}^{(5)^{2}}+10^{-3} s$,

$x_{n+1}^{(6)}=3.99 x_{n}^{(6)}\left(1-x_{n}^{(6)}\right)$,

$b_{n+1}^{(6)}=b_{n}^{(6)}+b_{n}^{(6)}\left(0.570-x_{n}^{(6)}\right)-10^{-3} z_{n}^{(6)^{2}}+10^{-3} s$,

$x_{n+1}^{(7)}=3.99 x_{n}^{(7)}\left(1-x_{n}^{(7)}\right)$,

$b_{n+1}^{(7)}=b_{n}^{(7)}+b_{n}^{(7)}\left(0.571-x_{n}^{(7)}\right)-10^{-3} z_{n}^{(7)^{2}}+10^{-3} s$,

$x_{n+1}^{(8)}=3.99 x_{n}^{(8)}\left(1-x_{n}^{(8)}\right)$,

$b_{n+1}^{(8)}=b_{n}^{(8)}+b_{n}^{(8)}\left(0.572-x_{n}^{(8)}\right)-10^{-3} z_{n}^{(8)^{2}}+10^{-3} s$,

$x_{n+1}^{(9)}=3.99 x_{n}^{(9)}\left(1-x_{n}^{(9)}\right)$,

$b_{n+1}^{(9)}=b_{n}^{(9)}+b_{n}^{(9)}\left(0.573-x_{n}^{(9)}\right)-10^{-3} z_{n}^{(9)^{2}}+10^{-3} s$,

$x_{n+1}^{(10)}=3.99 x_{n}^{(10)}\left(1-x_{n}^{(10)}\right)$,

$b_{n+1}^{(10)}=b_{n}^{(10)}+b_{n}^{(10)}\left(0.574-x_{n}^{(10)}\right)-10^{-3} z_{n}^{(10)^{2}}+10^{-3} s$,

$x_{n+1}^{(11)}=3.99 x_{n}^{(11)}\left(1-x_{n}^{(11)}\right)$,

$b_{n+1}^{(11)}=b_{n}^{(11)}+b_{n}^{(11)}\left(0.575-x_{n}^{(11)}\right)-10^{-3} z_{n}^{(11)^{2}}+10^{-3} s$,

$x_{n+1}^{(12)}=3.99 x_{n}^{(12)}\left(1-x_{n}^{(12)}\right)$,

$b_{n+1}^{(12)}=b_{n}^{(12)}+b_{n}^{(12)}\left(0.576-x_{n}^{(12)}\right)-10^{-3} z_{n}^{(12)^{2}}+10^{-3} s$,

$x_{n+1}^{(13)}=3.99 x_{n}^{(13)}\left(1-x_{n}^{(13)}\right)$,

$b_{n+1}^{(13)}=b_{n}^{(13)}+b_{n}^{(13)}\left(0.577-x_{n}^{(13)}\right)-10^{-3} z_{n}^{(13)^{2}}+10^{-3} s$,

$x_{n+1}^{(14)}=3.99 x_{n}^{(14)}\left(1-x_{n}^{(14)}\right)$,

$b_{n+1}^{(14)}=b_{n}^{(14)}+b_{n}^{(14)}\left(0.578-x_{n}^{(14)}\right)-10^{-3} z_{n}^{(14)^{2}}+10^{-3} s$,

$x_{n+1}^{(15)}=3.99 x_{n}^{(15)}\left(1-x_{n}^{(15)}\right)$,

$b_{n+1}^{(15)}=b_{n}^{(15)}+b_{n}^{(15)}\left(0.579-x_{n}^{(15)}\right)-10^{-3} z_{n}^{(15)^{2}}+10^{-3} s$,

$x_{n+1}^{(16)}=3.99 x_{n}^{(16)}\left(1-x_{n}^{(16)}\right)$,

$b_{n+1}^{(16)}=b_{n}^{(16)}+b_{n}^{(16)}\left(0.580-x_{n}^{(16)}\right)-10^{-3} z_{n}^{(16)^{2}}+10^{-3} s$,

$x_{n+1}^{(17)}=3.99 x_{n}^{(17)}\left(1-x_{n}^{(17)}\right)$,

$b_{n+1}^{(17)}=b_{n}^{(17)}+b_{n}^{(17)}\left(0.581-x_{n}^{(17)}\right)-10^{-3} z_{n}^{(17)^{2}}+10^{-3} s$,

$b_{n+1}^{(18)}=b_{n}^{(18)}+b_{n}^{(18)}\left(0.582-x_{n}^{(18)}\right)-10^{-3} z_{n}^{(18)^{2}}+10^{-3} s$,

$s_{n+1}=b_{n}^{(1)}+b_{n}^{(2)}+\cdots$.

Each "cell" (pair of variables with index ${ }^{(i)}, i=$ $1, \ldots, 18)$ involves a similar, formally identical, chaotic forcing variable. However, the initial conditions of the $x$-variables were different for each subsystem so that indeed the chaotic forcings are independent. Note also that each flare attractor
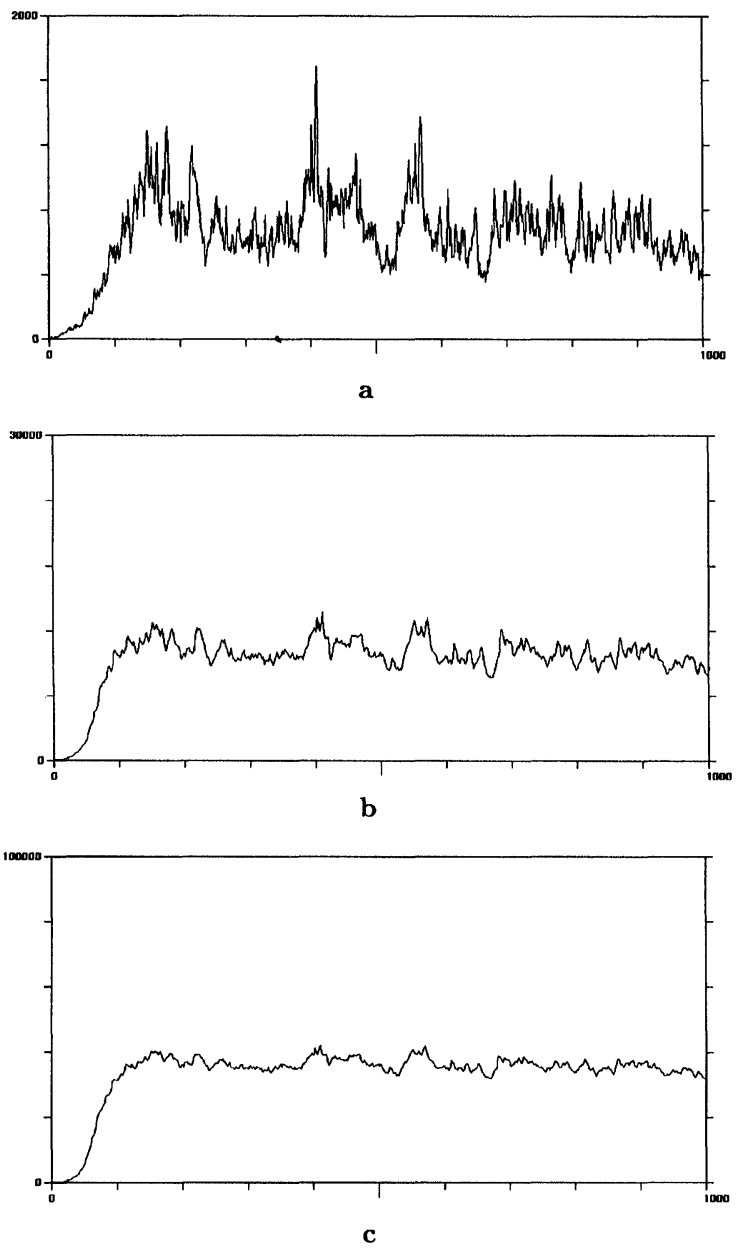

FIGURE 5 "Finite decay plot". Numerical simulation of Eq. (3), with the last line of Eq. (3) replaced by Eq. (4) indistinguishable from Fig. 4(d). (b) Medium smoothing $(a=0.2)$. (c) Strong smoothing $(a=0.05)$. Compare text. 
$\left(x^{(i)}, b^{(i)}\right)$ differs from its neighbors in that the value of the threshold used in the second variable $(b)$ is different in every case.

Figure 4(d), finally, shows the behavior of a single flare-attractor variable $\left(b^{(18)}\right)$, plotted against the sum variable, $s$, of all coupled flaring variables.

In the final picture, Fig. 5, we add some simulations in which the sum variable $(s)$ was made an integrator rather than being instantaneous as it was in Eq. (3). It now reads:

$$
s_{n+1}=s_{n}+b_{n}^{(1)}+b_{n}^{(2)}+\cdots-a s_{n} .
$$

The case with $a=1$ (instantaneous summing) has already been presented in Fig. 4(c). Figure 5 in addition shows three further cases with an increasingly strong smoothing-effect ( $a=0.99, a=0.2$, and $a=0.05$, respectively). Figure 5(b) to us looks a bit like a "frozen sea".

We present these last pictures in the hope that specialists dealing with realistic time series - like those taken from a real economic system like the stock market - may find some similarities between their own data and the sum signals of Fig. 5 generated by a "society" of flare attractors as it were.

\section{DISCUSSION}

The flare phenomenon is well-known from many natural situations like flaring outbursts from stars or irregularly erupting burning logs. The idea to consider "flaring" as a generic type of dynamical behavior was originally triggered by numerical experiments performed on Milnor-type attractors, cf. [9]. Milnor attractors [10] are in general (although not always) unbounded. An attractor at infinity and an attractor at zero (say) coexist in such a way that points in the intermediary region undecidably belong either to the one attractor's basin or to that of the other. This is called the "riddled basins" phenomenon, cf. [10,11,11a].

Flare attractors can be considered as "tamed" Milnor attractors. In Eq. (1), for example, a Milnor attractor is obtained if $\epsilon$ is put equal to zero. As soon as the flaring amplitude of a (non-generic) Milnor attractor is made bounded - for example, by introducing a growth limitation through assuming $\epsilon$ greater than zero however small - we have a (generic) flare attractor.

Flare attractors, in turn, belong into the class of Kaplan-Yorke attractors [12]. That is, they possess a small negative Lyapunov-characteristic exponent which is smaller in its numerical magnitude (closer to zero) than the positive LCE of the forcing chaos. This causes the Lyapunov-dimension of Kaplan-Yorke attractors to jump up by unity to resemble that of a hyperchaotic attractor (characterized by more than one positive LCE) [12]. Kaplan-Yorke attractors in general possess a nowhere-differentiable cross section on a Cantor set (that is, they belong into the class of singular-continuous-nowhere-differentiable attractors. [7,8]) The same features are inherited by the flaring-type Kaplan-Yorke attractors considered here. An example of a pertinent (nowhere-differentiable on a Cantor set) cross section has been presented in Fig. 2(c) above. An analytical study of a closely related map (lacking the last constant term in Eq. 2) appears possible.

The main question, in the present context, reads: Is there a connection to economics? The authors are painfully aware of the fact that they are not qualified to make an educated guess here. They were just struck by a recent reaction-diffusion model of an "evolutionary economy" proposed by Silverberg [13]. It both fit their intuitions and seemed to admit of a potential "enrichment" in terms of individually responding (not averaged over) economic units. This is where the flare attractor came to mind again as a potential stand-in for such a unit.

Real enterprises are, of course, much more complex than the here considered "units" (the flare attractors). Nevertheless there seemingly exists an intuitive connection: Friedrich Jahn's meteorlike rise and fall. Everbody knows about Wienerwald ${ }^{\circledR}$, the precursor to McDonald's ${ }^{\circledR}$ success story. Friedrich Jahn adhered to the domestic policy of putting every dollar earned into 
the next branch office of his chain, that is, the next Wienerwald "Stube". This fact in principle enables the occurrence of autocatalytic growth. A typical flare phenomenon followed - including the downfall of the empire. We recommend his autobiography, "Ein Leben für den Wienerwald - vom Kellner zum Millionär... und zurück" [14].

Jahn's sense of enterpreneurial management somehow resonates ("gibes") with our own intuition that a realistic economic subsystem is in general not completely immune to being governed by unpredictable symbolic-dynamics sequences [15]. To put this idea to a test, we came up with the above skeleton model of an economy. Other stochastic time inputs beside chaotic ones including hyperchaotic ones - can likewise be used numerically. In other words, the "flaring behavior" appears to be very robust indeed. For example, chemical reaction systems of the continuous type - so-called continuous-stirred-tank-reactors (CSTR's) - readily produce flaring behavior if autocatalytic subsystems with a threshold, analogous to the $b$ variable in Eq. (1) above, are introduced in the presence of a chaos-generating subsystem [9,16,17]. Flare attractors therefore appear to be robust constituents of many nonlinear dynamical systems with complex behavior including perhaps the economy, but including perhaps also a living cell.

To conclude, an important prototype of dynamical behavior may be hidden in everyday economic phenomena. We would like to invite criticism to our idea that it may be legitimate to believe that a four-variable continuous dynamics a three-variable chaotic attractor coupled to a threshold-type autocatalytic fourth variable, modelled in the simplest case by a discrete two-variable system like that of Eq. (1) - deserves to be elevated to the status of a new generic phenomenon. Is this phenomenon comparable in importance to chaos itself? At any rate, a new "module" in a nonlinear construction set appears to have been identified on a level slightly higher than the lowest-level single-variable modules that are so widely used today in simulation programs like Simulink ${ }^{\circledR}$, for example. Only the future can tell whether intermediate-level modelling approaches like the one proposed above have some practical usefulness.

\section{Acknowledgments}

We thank Vladimir Gontar, Michael Sonis, Peter Plath, Stephan Müller, Jürgen Parisi, Artur Schmidt, Jack Hudson, Thomas Kapitaniak, Jürgen Vollmer, Günther Radons, Peter Kloeden, Igor Gumowski, Joshua Epstein, Brian Arthur, Hans Guenther Stadlmayer, Gerald Silverberg, Frank Englmann and the late Richard Goodwin for discussions. For J. O. R.

\section{References}

[1] E.N. Lorenz (1969). Atmospheric predictability as revealed by naturally occurring analogies. J. Atmos. Sci., 26, 636.

[2] E. Lorenz (1993). The Essence of Chaos. University of Washington Press, Seattle.

[3] R.M. May (1976). Simple mathematical models with very complicated dynamics. Nature, 261, 459-467.

[4] H.T. Siegelmann (1995). Computation beyond the turing limit. Science, 268, 545-548.

[5] G.C. Hartmann and O.E. Rossler (1997). A self-similar flare attractor. In 4th Experimental Chaos Conference, Boca Raton, FL, August 6-8, 1997, (M. Ding and M. Shlesinger, eds.), Abstracts book, pp. 97-98.

[6] O.E. Rössler, R. Wais and R. Rössler (1992). Singularcontinuous Weierstrass-function attractors. In Proceedings of the 2nd International Conference on Fuzzy Logic \& Neural Networks, Iizuka, Japan, July 17-22, 1992, pp. 909-912.

[7] O.E. Rossler, C. Knudsen, J.L. Hudson and I. Tsuda (1995). Nowhere-differentiable attractors. International Journal of Intelligent Systems, 10, 15-23.

[8] O.E. Rossler (1993). Singular-continuous nowhere-differentiability in attractors. In A.E. Andersson, S.I. Andersson and U. Ottoson, eds., Dynamical Systems - Theory and Applications, pp. 205-232. World Scientific, Singapore.

[9] Georg Hartmann (1994). Attractors with flares in chemistry and nonlinear dynamics (in German). Diploma thesis in chemistry, University of Tübingen.

[10] J.C. Alexander, J.A. Yorke, Z. You and I. Kan (1992). Riddled basins. Intern. J. Bifurcation and Chaos, 2(4), 795-813.

[11] P. Beckmann (1996). Theoretical Foundation of the Nonlinear Dynamics of Dissipative Systems (in German). Lecture notes, University of Mainz, Germany (unpublished).

† "A life for the Wienerwald - from waiter to millionaire... and back". 
[11a] K. Kaneko (1994). Information cascade with marginal stability in a network of chaotic elements. Physica D, 77, $456-472$.

[12] J.L. Kaplan and J.A. Yorke (1979). Chaotic behavior of multidimensional difference equations. Lecture Notes in Mathematics, 730, 204-227.

[13] G. Silverberg and B. Verspagen (1995). Evolutionary theorizing on economic growth. In Kurt Dopfer, ed., The Evolutionary Principle of Economics. Kluwer Academic Publishers, Norwell, MA.

[14] Friedrich Jahn (1993). Ein Leben für den Wienerwald. Jahn Verlag.
[15] O.E. Rossler and G.C. Hartmann (1997). A society of flare attractors. In S.C. Müller, ed., Diffusion and Structure Formation, 18th WE-Heraeus Seminar, November 10-12, 1997, Bad Honnef, Abstracts book, p. 22.

[16] O.E. Rossler and G.C. Hartmann (1995). Attractors with flares. Fractals, 3, 285-296.

[17] G.C. Hartmann and O.E. Rossler (1995). Flaring - A new type of dynamical behaviour. In M.M. Novak, ed., Fractal Reviews in the Natural and Applied Sciences. Chapman \& Hall, London, pp. 372-376. 


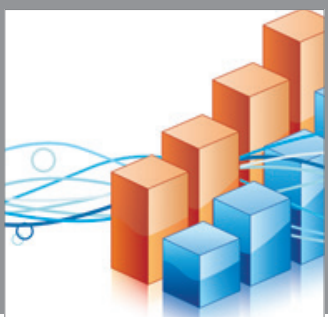

Advances in

Operations Research

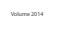

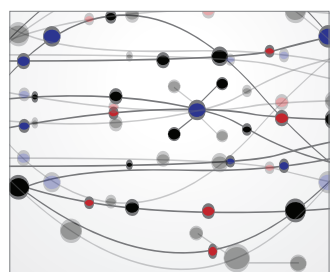

\section{The Scientific} World Journal
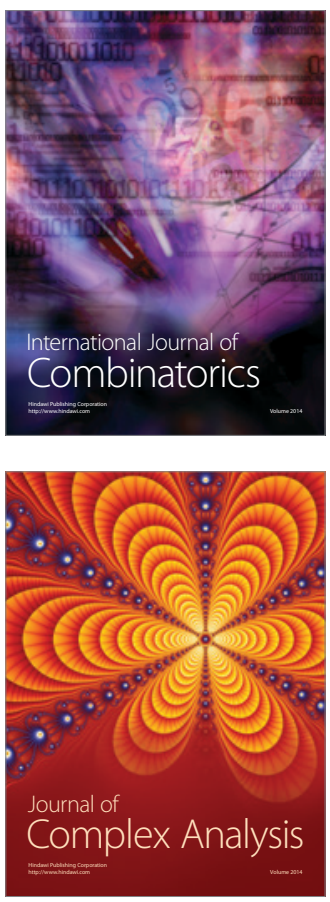

International Journal of

Mathematics and

Mathematical

Sciences
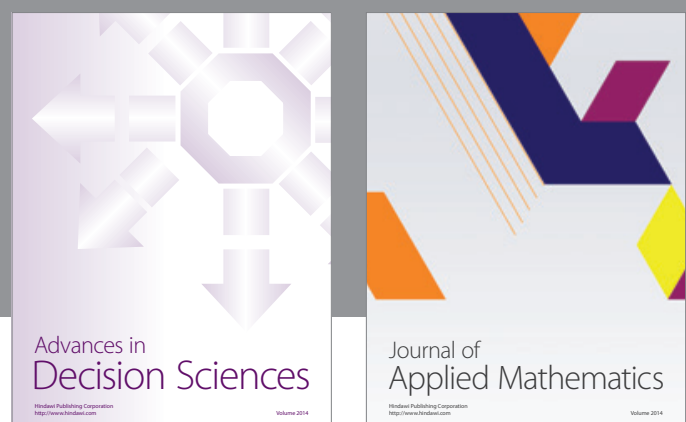

Journal of

Applied Mathematics
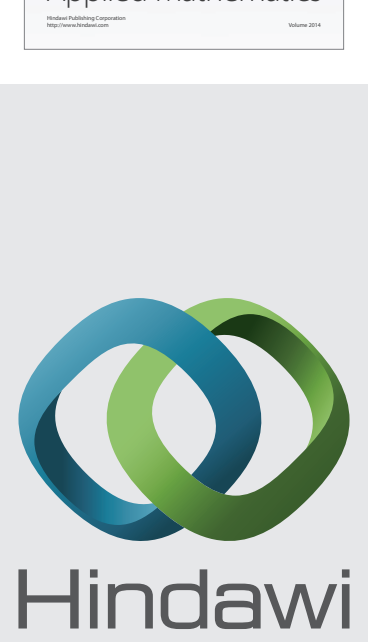

Submit your manuscripts at http://www.hindawi.com
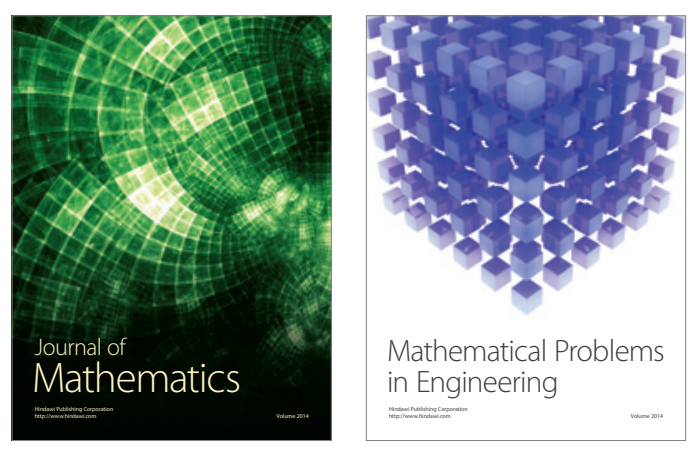

Mathematical Problems in Engineering
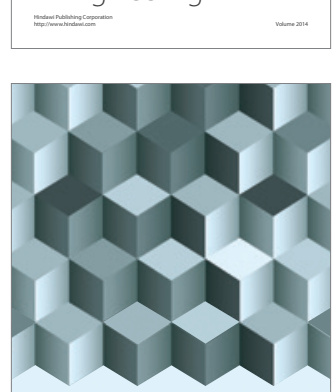

Journal of

Function Spaces
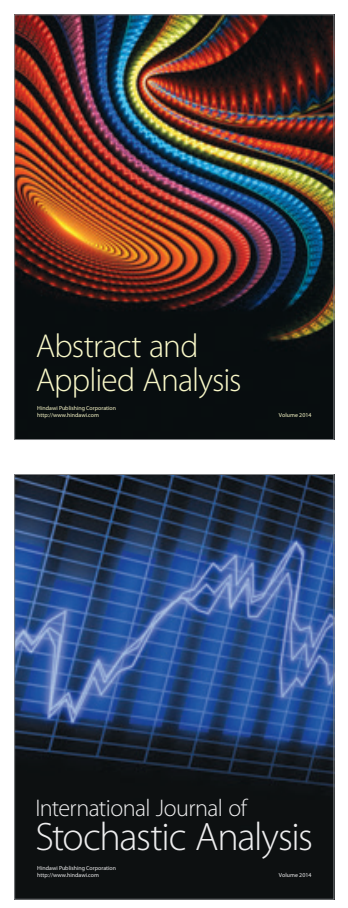

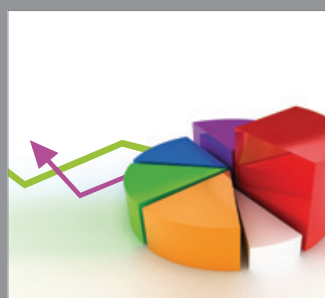

ournal of

Probability and Statistics

Promensencen
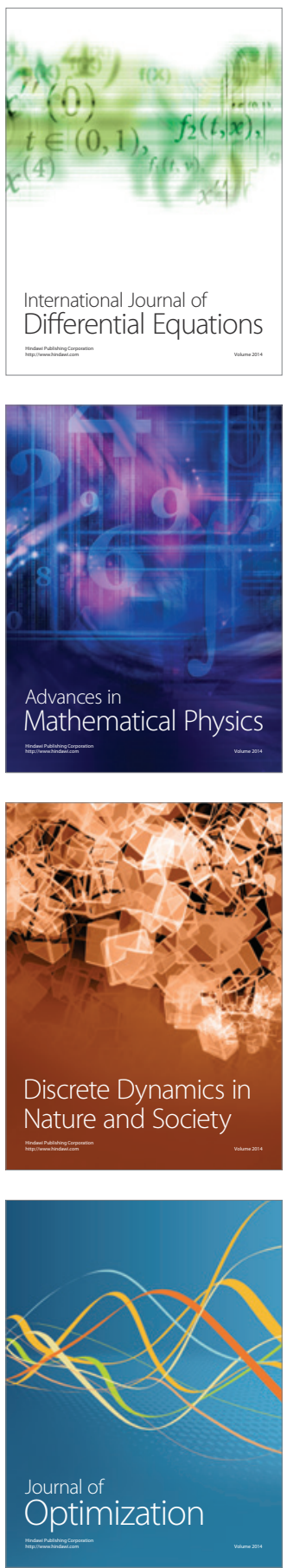